\title{
Las plantas medicinales como estrategia pedagógica para la conservación del Medio Ambiente ${ }^{1}$ Medicinal plants as a pedagogical strategy for the conservation of the Environment
}

DOI: http://dx.doi.org/10.17981/cultedusoc.9.2.2018.12

Fecha de recepción: 16/06/2018. Fecha de aceptación: 11/09/2018

\author{
Yeovanna Rangel-Ospino ${ }^{2}$ \\ Sandra Díaz-Santos y Reinaldo Guerrero-Gutiérrez \\ IED Arcesio Caliz Amador sede Principal (Colombia) \\ yeovaos75@hotmail.com
}

Para citar este artículo

Rangel-Ospino, Y., Díaz-Santos, S. y Guerrero-Gutiérrez, R. (2018). Las plantas medicinales como estrategia pedagógica para la conservación del Medio Ambiente. Cultura. Educación y Sociedad 9(2), 129-136. DOI: http://dx.doi.org/10.17981/cultedusoc.9.2.2018.12

\section{Resumen}

Las plantas medicinales se han utilizado desde la antigüedad para tratar dolencias en los seres humanos pero la mayor cantidad de información sobre el uso de esta medicina alternativa la poseen los adultos mayores. El objetivo de la presente investigación entonces es identificar las plantas medicinales de la región para incentivar el uso curativo y promover la conservación medioambiental. Se identifican las fuentes de conocimiento y finalmente se difunde e incentiva al uso de las plantas medicinales de la región para el tratamiento de las enfermedades. La investigación es de tipo cuantitativa, de alcance descriptivo. Se trabajó con los estudiantes de la IED Arcesio Cáliz Amador. A modo de conclusión es importante resaltar que los estudiantes distinguen las plantas medicinales, tienen conocimientos sobre su uso, sin embargo, requieren de mayor formación frente al tema.

Palabras clave: Plantas medicinales, estrategia pedagógica, medicina alternativa, enfermedades, medio ambiente.

\begin{abstract}
Medicinal plants have been used since ancient times to treat ailments in humans but the greatest amount of information on the use of this alternative medicine is possessed by the elderly. The objective of the present investigation then is to identify the medicinal plants of the region to encourage the curative use and promote environmental conservation. The sources of knowledge are identified and finally the use of medicinal plants in the region for the treatment of diseases is disseminated and encouraged. The research is quantitative, descriptive in scope. We worked with the students of the Ides Arcesio Cáliz Amador. By way of conclusion it is important to highlight that students distinguish medicinal plants, have knowledge about their use, however, they require more training on the subject.
\end{abstract}

Keywords: Medicinal plants, pedagogical strategy, alternative medicine, diseases, environment.

\footnotetext{
${ }^{1}$ Este artículo ha sido derivado del Programa de Fortalecimiento de la Cultura Ciudadana y Democrática CT+I a través de la IEP apoyada en TIC en el Departamento de Magdalena: CICLÓN. Desarrollado con docentes miembros del Grupo de investigación "Investigadores Botánicos" pertenecientes a la Institución Educativa Departamental Arcesio Caliz Amador sede Principal.

${ }^{2}$ Líder del grupo de investigación "Investigadores Botánicos".
} 


\section{Introducción}

Las plantas medicinales se han utilizado desde la antigüedad para tratar dolencias en los seres humanos pero la mayor cantidad de información sobre el uso de esta medicina alternativa la poseen los adultos mayores por lo que esta información se está perdiendo o a punto de extinguirse en la región debido a que las nuevas generaciones están poco interesadas en aprender sobre esta temática interrumpiéndose la transmisión de conocimientos. Por tal motivo el proyecto de investigación se enfocado en identificar y rescatar el uso de las plantas medicinales lo que permitirá conservar la información sobre las plantas medicinales más utilizadas y como contribuir a la protección del medio ambiente en el Municipio del Banco Magdalena.

Es desde que existe la sociedad, que hay preocupación por el uso de los recursos naturales, y el cuidado y mantenimiento del planeta se hace cada vez más una propuesta que no se debe prolongar. Con el paso de los años se ha notado fuertemente un accionar despreocupado, egoísta y contaminante por parte del hombre sobre los recursos, sin embargo; actualmente se ha hecho consciente que los daños causados afectan directamente la el accionar y las relaciones humanas. Es por esto que se puede inferir que los pensamientos sociales, culturales, económicos han permitido trascender de conductas que anteriormente dominaban el comportamiento de los individuos. (Herrera, Acuña \& Gil; 2014).

Según PNUMA. (1990). Los cambios actuales han exigido lograr grandes desarrollos en materia ambiental, pues el humano amenaza con perjudicar el ambiente más rápido de lo que este puede regenerarse; toma importancia entonces la identificación, la comprensión e intervención de las variables psicológicas implicadas en el cuidado del medioambiente, para que sean reflejadas en el ámbito conductual.
Es importante científica y académicamente comprender el papel de los factores conductuales y mentales implicados en la relación del hombre con la naturaleza es esencial para encontrar la solución al problema medioambiental (Ramírez \& Del La Hoz; 2017, citando a Bravo, 2010), ya que se necesita conocer como asumen los humanos el problema ambiental para reducirlo desde sus inicios y no buscar soluciones cuando ya está establecido, es decir enfocarse en un primer momento en la reducción, pero principalmente en la promoción y prevención; así como buscar cambiar esa posición egoísta y antropocéntrica que asume el hombre frente a su entorno.

Esta información sería absolutamente útil para crear modelos enfocados a explicar por qué y de qué manera las personas realizan determinadas conductas en relación con el ambiente, además de diseñar modelos explicativos de comportamientos ambientales dentro de la empresa y cómo son asumidos por los trabajadores. (Ramírez \& Del La Hoz; 2017).

Los problemas medioambientales que dan origen a la presente investigación buscan dejar ver cómo están las relaciones e interacciones del organismo y el ambiente, y que se permita comprender el impacto que tiene el ambiente sobre la persona, y el impacto que tiene la persona sobre su ambiente. De esta manera, se plantea la necesidad de realizar cambios actitudinales en los seres humanos, que conduzcan a la generación de modelos y patrones de conducta más acordes con el cuidado del Medio Ambiente partiendo desde su ciclo de interacción vital, a pesar de que autores como González (2005), afirman que la atribución de la degradación ambiental se externaliza al contexto global, sin contemplar la experiencia personal como parte del compromiso en el fortalecimiento de conductas pro ambientales (Herrera, 2014). 
Según Herrera, 2014), actualmente se está teniendo en cuenta un modelo para el estudio de la relación de los valores personales con el comportamiento pro ambiental es el de "La estructura y contenido universal de los valores humanos". En él se clasifican una serie de contenidos que comparten los valores, a manera de objetivos que motivan a la acción, los cuales se orientan bajo dos dimensiones: Conservadurismo (seguridad, conformidad, tradición) Vs apertura al cambio (autodirección, hedonismo, estimulación) y autotrascendencia (benevolencia, universalismo) Vs autopromoción (logros, poder) (Schwartz, 1992).

\section{Conceptualización de las plantas medicinales}

Las Plantas Medicinales, conocida también como "Medicina Alternativa", es una droga vegetal que ofrece la naturaleza, para la elaboración de diversos remedios como: infusiones, ungüento, jarabe, comprimidos, cremas, cápsulas, elixir, entre otros, para el tratamiento de alguna malestar y que se encuentra en el mercado a costos bajos y un gran porcentaje de la población del Banco Magdalena y sus alrededores. Según los ancestros en los tiempos antiguos ante cualquier dolencia tiene casi siempre como primera opción algún remedio o medicamento natural antes de acudir a un médico o centro hospitalario, esto ha cambiado un poco con el crecimiento acelerado de las sociedades. (Arango, 2004).

Desde luego medicina alternativa no debe sustituir los conceptos médicos, es utilizada mientras se acude a los servicios hospitalarios o científico. Los adultos mayores son los que poseen gran información poseen acerca del uso de las plantas medicinales en nuestra región por lo se hace necesario recopilar esa información sobre las experiencias de nuestros abuelos para fortalecer la existente en libros y diferen- tes fuentes informativas a través de un blog sobre medicina natural para acceder a ella de manera rápida y eficaz. (Arango, 2004).

Es en los pueblos que gran parte de la población aún emplea tradicionalmente la variedad de plantas medicinales para combatir las enfermedades, no obstante, la investigación de orden científico sobre este campo $n$ ha tenido gran acogida por parte del estado.

Aunque internacionalmente se cuenta con una normatividad que deja ver a las plantas medicinales como insumo medicinal, de acuerdo a las tradiciones indígenas, se necesita de mayor promoción y fortalecimiento de investigaciones frente a los múltiples beneficios para el tratamiento de enfermedades y asi poder enfrentar el sinnúmero de problemas de orden sanitario. Lo anterior deja ver la baja fuerza científica y de innovación con la que se cuenta frente a esta línea, lo que genera escases de investigaciones sobre el tema. (Cosme, 2008).

Según Cosme, (2008). "La medicina como una forma de preservar la salud y la vida misma de las personas se ha visto desarrollada de muchas formas, tal es el caso de las primeras poblaciones de la nación colombiana que practicaban el uso de plantas medicinales como forma de curar enfermedades y preservar la salud".

Los descendientes de las primeras comunidades indígenas y de africanos en el país son los que se caracterizan por el masivo cultivo y consumo de plantas medicinales, esto logrando conservar las costumbres tradicionales y culturales de sus antepasados, un ejemplo de esto es la comunidad indígena Yukpa que utilizan en la actualidad las plantas con fines medicinales, considerándola parte de su identidad y cultura, trascendiendo de la población mencionada y llegando a las zonas urbanas, siendo las casas naturistas un caso ejemplar (Sauvé, 1999; Novo, 2009). 
Aún no se tiene caro conocimiento sobre las consecuencias que pueden generar los productos medicinales en la salud de los individuos. Por lo general puede que estos medicamentes brinden una mejora rápida de algún tipo de enfermedad, sin embargo, casos demuestran que pueden existir efectos secundarios con el pasar del tiempo. Por tal motivo es que se busca promover el estudio de recetas y preparación de los remedios derivados de las plantas medicinales como medio de solución (Escamilla, y Moreno. 2015).

Cabe resaltar nuevamente que las medicinas llamadas alternativas no puede reemplazar los criterios médicos, por considerar que no es prudente ni sensato debido a que un síntoma puede significar el inicio de varias enfermedades o una muy grave ,y solo un médico apoyado en los avances de la ciencia puede determinar procedimientos adecuados para reestablecer oportunamente la salud (Linares, 2013).

El uso de las plantas medicinales, no solo se convierten en una alternativa de conservación del medio ambiente, por el contrario, dependiendo de los intereses y el trabajo colaborativo puede lograrse generar una demanda de consumo traspasar a lo que hoy se conoce como Marketing Educativo, "creando y facilitando que los clientes consumidores (estudiantes), puedan interactuar en situaciones de aprendizaje participativo dentro y fuera de contextos educativos, a partir de entender que enseñar supone reconocer la individualidad y la diversidad de los alumnos, en la convicción de que el aprendizaje es un proceso vivencial, fruto de la reflexión, la discusión, el análisis, la interacción y retroalimentación grupal". (Sanz, \& Crissien, García, \& Patiño. 2017).

Según Escamilla, y Moreno. (2015). Es la medicina de la modernidad la que permite ir remplazando la medicina antigua, es decir el uso de hierbas y contenidos bajo la ritualidad, pero no de todo puesto que los adultos tardíos confían en lo que desde la historia han utilizado.

El objetivo principal entonces, es dar a conocer a la comunidad estudiantil las propiedades curativas de las plantas medicinales para tratar diversas enfermedades y conservar una buena salud. Las personas de todos los estratos sociales especialmente los bajos utilizan por excelencia las plantas para curar sus enfermedades, realizando los procedimientos por cuenta propia o apoyándose de expertos que en un precio muy bajo cobran por facilitar el remedio.

Existe una probabilidad alta que algunas personas puedan ser engañadas 0 utilizadas para la demanda de consumo de los productos medicinales, por lo cual se debe contar con apoyo científico, y ante una enfermedad consultas con personas especializadas y formadas en medicina recomendando que se tenga en cuenta la medicina naturista pero no como opción principal.

Las plantas medicinales son aquellas que contienen principios que pueden utilizarse en la curación de enfermedades. Desde la época prehistórica en la mayoría de las culturas conocidas, el hombre ha experimentado con diferentes partes de hierbas, arbustos y árboles. El primer heterósido que se descubrió fue la salicina (extraído de Salix alba) principal componente de la aspirina comercial.
"Se calcula en unas 260.000 las espe- cies de plantas que se conocen en la ac- tualidad, de las que el $10 \%$ se pueden considerar medicinales, es decir, se encuentran recogidas en los tratados médicos de fitoterapia, modernos y de épocas pasadas, por presentar algún uso. La fitoterapia (uso de plantas medicinales) tiene sus orígenes en los albores de la humanidad, desde que aparecen registros o referencias fiables". (Cruz, 2007). 
El consumo de plantas medicinales ha aumentado a nivel mundial en lo últimos tiempos, logrando integrarlos con medicamentos asignados por los médicos. Ex de poca veracidad partir de que los productos naturales tienen todas las ventajas posibles requiriendo necesariamente de revisiones científicas para la emisión de diagnósticos confiables.

Fines de utilidad de las plantas medicinales. (Fundación Salud y Naturaleza. 2007).

Aliviar dolores e irritación de la garganta combatir parásitos

Inflamación intestinal combatir el mal aliento

Para la tos Trastorno del aparato respiratorio

Dolores menstruales Previene el estreñimiento

Fuente: Elaboración propia. (2018)

Plantas medicinales como estrategia pedagógica

Según el Ministerio De Educación Nacional (2014) el objetivo primordial es mejorar la calidad educativa, y para ello han diseñado programas que buscan la transformación de los escenarios en donde se propende la enseñanza- aprendizaje.

Los programas pedagógicos sirven como material de apoyo en los procesos de enseñanza, que según el Ministerio de Educación de Chile (2008) "Tiene como propósito facilitar y operacionalizar la implementación de las Bases Curriculares de la Educación.”(p. 12).

La ejecución de estrategias pedagógicas permite hacer seguimiento y gradación a partir de la observación a los aprendizajes para la formación de los estudiantes desde lo más simple hasta lo más complejo. Uno de los propósito primordiales es adecuar de forma coherente las oportunidades educativas, que no solo promueven la enseñanza, sino además que potencian las capacidades del educador.
Es importante mencionar que para llevar a cabo un programa pedagógico según Guerrero(2016)se debe tener en cuenta varios aspectos: La organización, la distribución temporal de los aprendizajes, las alternativas de planificación para concretar lo que se pretende alcanzar, la oportunidad de poder incluir nuevos aprendizajes, la adaptación de las experiencias, la selección de las mismas, la pertinencia de la didáctica, los procedimientos para ejecutar los seguimientos a los aprendizajes y la comunicación de avances y resultados, propios del objeto de interés y que fundamentan todas la operacionalizacion curricular.

Todo programa pedagógico propiamente dicho parte de unas necesidades curriculares, las cuales promueven el trabajo cooperativo entre estudiantes y docentes, fortaleciendo la observación y el análisis de los aprendizajes con el propósito de organizar una enseñanza mar contextualizada. Por tal motivo el uso de las plantas medicinales, alternada a las necesidades de la comunidad educativa, busca articular a los procesos de enseñanza nuevas formas vivenciales que motiven al estudiante a aprender, y a construir conocimiento a raíz de la práctica.

\section{Metodología}

La investigación es de tipo cuantitativa, bajo el paradigma empírico-analítico con un alcance descriptivo bajo un diseño no experimental. Se estudian los fenómenos en su contexto natural, sin manipular las variables.

\section{Población y muestra}

La población participante de la investigación son estudiantes de la Institución Educativa Departamental Arcesio Cáliz Amador de El Banco Magdalena con una muestra de 23 alumnos de la jornada de la mañana. 
Técnica e instrumento de recolección de información

De acuerdo a las características del escenario de investigación se decide utilizar como técnicas de recolección: La encuesta y como instrumento el cuestionario.

\section{Procedimiento}

La información es registrada y posteriormente tabulada permitiendo su clasificación y ofreciendo un insumo para el análisis de datos. Se construyeron los contenidos de acuerdo al fenómeno estudiado. La encuesta fue enviada a pares expertos para su respectiva validación. Una vez validados aplicó una prueba piloto, tomando los aportes más significativos de los aspectos evaluados.

El diseño de las preguntas, tuvo una guía que permitiera elaboración definitiva de la encuesta teniendo en cuenta las siguientes variables: Manejo del tema, reconocimiento de las plantas de la región, beneficios y medios de consulta.

Culminada la prueba piloto se envió de nuevo a los jueces expertos, quienes hicieron las correcciones y recomendaciones pertinentes. Finalmente se procede a realizar la aplicación a la población objeto de estudio.

\section{Resultados}

A continuación, se presentas los resultados derivados de la encuesta aplicada a los estudiantes.
Manejo del tema: ¿Qué son las plantas medicinales?

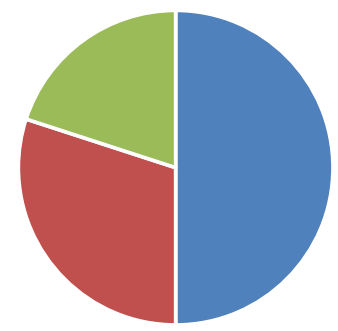

- Remedios de la Naturaleza

- Medios naturales curativos

- Utilizados para mejorar la salud .

Primera variable: Manejo del tema.

En el análisis de los resultados de las entrevistas realizadas a los estudiantes, $\mathrm{y}$ de los aspectos que estos manifiestan en torno al interrogante Para usted ¿Qué son las plantas medicinales? El 50\% de los encuestados respondió que son remedios de la naturaleza, el 30\% restante manifestó que son medios naturales curativos y el $20 \%$ expresó que se utilizan para mejorar la salud.

Reconocimiento de las plantas: ¿Qué plantas medicinales conoces?

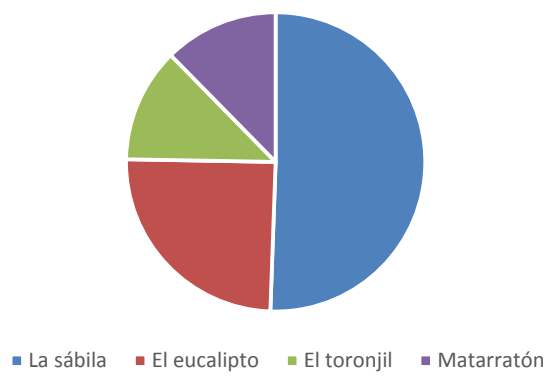

\section{Segunda variable:}

Reconocimiento de las plantas.

En el análisis de los resultados al siguiente ¿Qué plantas medicinales conoces? se pudo comprobar que el $45 \%$ reconoce la sábila, el 11\% el eucalipto, el $22 \%$ el toronjil, el $11 \%$ matarratón y el $11 \%$ el totumo. 
Beneficios: ¿Beneficio de utilizar las plantas medicinales?

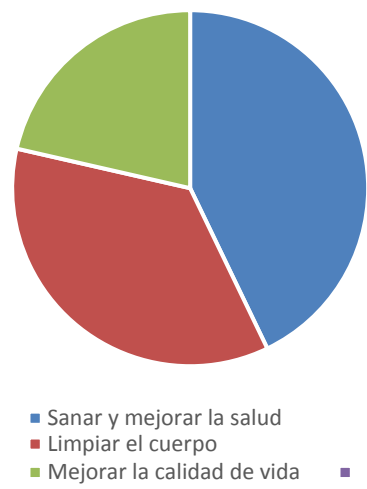

Tercera variable: Beneficios.

Las respuestas dadas por los estudiantes sobre el interrogante ¿Cuáles son los beneficios de utilizar las plantas medicinales? El 30\% expresó sirven para sanar y para mejorar la salud, el $25 \%$ para limpiar el cuerpo y $15 \%$ para mejorar la calidad de vida. Con este interrogante los estudiantes reconocen sobre la importancia de las plantas medicinales para la salud del hombre.

Medio de cosulta:

¿Através de que medio te gustó conocer sobre el uso de las plantas medicinales?

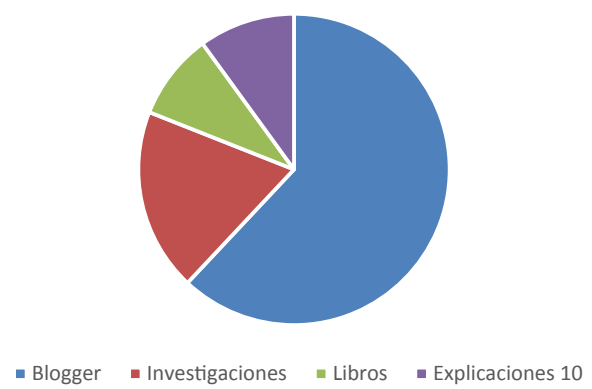

Cuarta variable: Medio de consulta.

Los entrevistados respondieron al interrogante ¿A través de que medio te gustó conocer sobre el uso de las plantas medicinales? El $62 \%$ respondió que le gustaría conocer más del tema a través de blogger, el $19 \%$ a través de investigaciones, el $10 \%$ explicaciones y el $9 \%$ consultando en libros.

\section{Conclusiones}

Con la información recolectada, y los resultados arrojados se establecen establecer las siguientes conclusiones:

- Es importante resaltar que en el plan de área del grado $7^{\circ}$ se trabaja con temáticas sobre las plantas, donde el docente de ciencias naturales a través de estrategias lúdico pedagógicas se orientaron procesos de adquisición de conocimientos sobre el tema, de esta forma los estudiantes se motivaron en participar activamente.

- Con el desarrollo de estas actividades los estudiantes amplían su conocimiento sobre las plantas con que cuentan para la solución de sanación de ciertas enfermedades, sin afectar el organismo, es decir, sin contar indicaciones. Como también reconocieron los cuidados que se debe tener en el uso de estas plantas.

- los estudiantes identifican los beneficios al utilizar plantas medicinales para curar enfermedad y evitar el sufrimiento de la mente y el cuerpo.

Uno de los mayores valores que ofrecen las TIC es la posibilidad de crear entornos que potencien la transferencia información y la comunicación entre el estudiante y el profesor o entre el propio estudiante, lo cual puede ser aprovechado para mejorar el aprendizaje. Por todo lo anterior este proceso de investigación pretende recopilar toda la información sobre las plantas medicinales de la región para luego sean divulgadas.

Como estrategia educativa la divulgación a través de medios digitales permitiendo a alumnado transmitir de una manera significativa sus vivencias educativas, promoviendo además competencias digitales, y por ende mejorando el proceso de enseñanza aprendizaje. 


\section{Referencias}

Acuña, M. y Gil, L. (2014). Descripción de los factores motivacionales en el comportamiento de consumo de telefonía móvil en estudiantes universitarios. (Tesis de Pregrado). Barranquilla, Colombia.

Arango, S. (2004). Guía de plantas medicinales de uso común en Salento Colombia. Missouri Botánica Garden. Documento recuperado de: http://www. mobot.org/plantscience/ccsd/Publication\%20Articles/GuiaMedicinales.pdf

Cosme, I. (2008). El uso de las plantas medicinales. Revista Intercultural. Documento recuperado de: https://cdigital. uv.mx/bitstream/123456789/8921/1/ tra6_p23-26_2010-0.pdf

Cruz, J. (2007). Más de cien plantas medicinales. Perz Galdós. Documento recuperado de: http://www.lacasadelvolcan.es/DOCUMENTOS/100PM.pdf.

Escamilla, B. y Moreno, P. (2015). Plantas medicinales. Primera edición. México. Documento recuperado de: http:// www.itto.int/files/itto_project_db_input/3000/Technical/Manual\%20plantas\%20medicinales.pdf

Fundación Salud y Naturaleza. (2007). Libro blanco de los herbolarios y las plantas medicinales. Papaver rhoeas L. Documento recuperado de: https://www.fitoterapia.net/ archivos/200701/260307libro-2.pdf?1

Fundación Secretos para Contar. (2014). Los secretos de las plantas 50 plantas medicinales en su huerta. Secretos para contar. Tercera Edición. Documento recuperado de: http://libros. secretosparacontar.org/wpcontent/ uploads/2015/04/los_secretos_de_las_ platas.pdf
Herrera, K., Ramirez, M. y De la Hoz, M. (2015). Relación entre las prácticas ambientales y el modelo de la conducta planificada, en los trabajadores de una empresa de insumos químicos en la ciudad de Barranquilla. (Tesis de Pregrado). Barranquilla, Colombia.

Herrera, K. y Bravo, E. (2013). Perspectiva de la ecología en la comprensión de los comportamientos ambientales En: Venezuela Omnia 1315-8856 Universidad Del Zulia.

Linares, N. (2013). La farmacia de la Naturaleza. UPA Madrid. Documento recuperado de: http://www.fademur. es/_documentos/ponencias/Ponencia_ Fademur_farmacia_OK.pdf

Sanz, D., Crissien, T., García, J. y Patiño, M. (2017). Marketing educativo como estrategia de negocio en universidades Privadas. En Desarrollo Gerencial Revista de la Facultad de Ciencias Económicas Administrativas y Contables de la Universidad Simón Bolivar-Colombia, 9(1), 160-175.

Sauvé, L. (1999). La educación ambiental entre la modernidad y la posmodernidad: En busca de un marco educativo de referencia integrador. Tópicos. Vol. 1. (2). México.

Novo, M. (2009). La educación ambiental, una genuina educación para el desarrollo sostenible. Revista de Educación. Universidad Nacional de Educación a Distancia (UNED). Madrid, España.

PNUMA. (1990). Educación ambiental: modulo para la formación de profesores de ciencias y de supervisores para escuelas secundarias. Orelac. Santiago de Chile. Documento recuperado de: http://unesdoc.unesco.org/ images/0007/000714/071480so.pdf 